\title{
Absorbable scaffolds noninferior to metallic stents at 1 year
}

In a randomized trial conducted in Japan, a drug-eluting bioresorbable vascular scaffold (BVS) has shown medium-term noninferiority to a metallic drugeluting stent (DES) in both clinical and angiographic outcomes for percutaneous coronary intervention (PCI) in patients with coronary artery disease (CAD).

BVSs provide mediumterm physical support and antiproliferative-drug delivery for treatment of vascular stenosis. Unlike traditional metallic stents, BVSs are eventually absorbed, and evidence suggests they are associated with favourable vascular responses in the long term. However, the short-term and medium-term safety and efficacy profiles of these scaffolds, although important, are currently undefined.

ABSORB Japan, the results of which were presented at the ESC Congress 2015 and published in the European Heart Journal, was a single-blind, multicentre trial in which 400 patients with lesions in epicardial vessels were randomly assigned in a 2:1 ratio to $\mathrm{PCI}$ with a BVS or a cobaltchromium everolimuseluting DES. The primary end point of the trial, measured at 12 months, was target-lesion failure, a composite of cardiac death, myocardial infarction attributable to the target vessel, and the need for ischaemia-driven target-lesion revascularization. A secondary end point, measured at 13 months, was angiographic in-segment late lumen loss, a measure of restenosis.

Mean patient age was 67.2 years, $36.0 \%$ had diabetes mellitus, and all had evidence of ischaemia, with stable CAD in $88.0 \%$. Successful pre-dilatation of target lesions was mandatory. The rates of successful deployment of the two devices were similar.

At 12 months, the rates of occurrence of target-lesion failure were $4.2 \%$ and $3.8 \%$ in patients treated with BVS and DES devices, respectively, demonstrating the noninferiority of the BVS

$(P<0.0001)$. No significant differences were seen between the devices for individual end points. At 13 months, angiographic follow-up showed in-segment late lumen loss of $0.13 \pm 0.30 \mathrm{~mm}$ for BVS, compared with $0.12 \pm 0.32 \mathrm{~mm}$ for DES devices, again demonstrating noninferiority of the BVS $(P<0.0001)$.

The performance of a BVS depends upon a number of factors, including physical properties of the device and procedural variables such as post-implantation dilatation. The accumulation of clinical experience with BVS devices should enable optimization of procedures, and the development of the next generation of BVSs could further improve outcomes.

The selective nature of the patient population in this study means that the "results should not be generalized to complex lesions, which are often encountered in clinical practice, such as bifurcations, heavily calcified lesions, diffuse disease, and thrombus," say the researchers. Nonetheless, the results "support the feasibility of BVS use to potentially improve the long-term outcomes of patients undergoing PCI".

\section{Robert Phillips}

Original article Kimura, T. et al. A randomized
trial evaluating everolimus-eluting Absorb
bioresorbable scaffolds vs. everolimus-eluting
metallic stents in patients with coronary
artery disease: ABSORB Japan. Eur. Heart J.
doi:10.1093/eurheartj/ehv435

